\title{
Measurement of Rotorcraft Blade Deformation using Projection Moiré Interferometry
}

\author{
Gary A. Fleming \\ National Aeronautics and Space Administration \\ Langley Research Center \\ Hampton, Virginia 23681-0001, USA
}

and

Susan Althoff Gorton

U.S. Army Aeroflightdynamics Directorate

Hampton, Virginia 23681-0001, USA

\begin{abstract}
Projection Moiré Interferometry (PMI) has been used to obtain near instantaneous, quantitative blade deformation measurements of a generic rotorcraft model at several test conditions. These laser-based measurements provide quantitative, whole field, dynamic blade deformation profiles conditionally sampled as a function of rotor azimuth. The instantaneous nature of the measurements permits computation of the mean and unsteady blade deformation, blade bending, and twist. The PMI method is presented, and the image processing steps required to obtain quantitative deformation profiles from PMI interferograms are described. Experimental results are provided which show blade bending, twist, and unsteady motion. This initial proof-of-concept test has demonstrated the capability of PMI to acquire accurate, full field rotorcraft blade deformation data.
\end{abstract}

Keywords: Projection Moiré Interferometry, PMI, rotorcraft, helicopter, blade, deformation, deflection

\section{INTRODUCTION}

Rotorcraft blade dynamics are a driving factor in the design of low noise, low vibration helicopters. Blade geometry, stiffness, and structural response significantly affect rotor performance and acoustics. The development of dynamic prediction codes has been hampered by the lack of experimental data, due in part to inadequate instrumentation. The instrumentation required to measure the mean and fluctuating components of blade deformation and attitude are subject to many restrictions dictated by the complexities of the spinning rotor. In general, the instrumentation must be: (a) noncontacting to avoid artificially induced mechanical and aerodynamic effects; (b) capable of acquiring near-instantaneous measurements at designated azimuth positions; and (c) capable of obtaining measurements at multiple spanwise and chordwise locations along the blade. Instrumentation satisfying these criteria and their application to rotorcraft blade dynamics has just begun to emerge. As part of the recent Higher harmonic control Aeroacoustic Rotor Test (HART), Dutch, German, and French researchers conducted comparative testing of three different blade deflection methods in the German-Dutch Wind Tunnel (DNW) ${ }^{1}$. Methods evaluated were (a) conventional strain gauge, (b) Target Attitude in Real Time (TART), and (c) projected grid method. Blade deflections measured using these techniques were in good agreement, but advantages were noted for the projected grid method. A technique similar to the projected grid method, Projection Moiré Interferometry (PMI), has recently been applied in the NASA - Langley 14-by 22-Foot Subsonic Wind Tunnel to measure mean and fluctuating blade deformation profiles. PMI differs from the projected grid method in that PMI uses interferometric methods to extract quantitative deformation data from the raw images, whereas the projected grid method uses grid line registration and tracking. This paper describes the PMI technique, image processing steps, and its application to measuring azimuth dependent mean and fluctuating blade deformation profiles. Blade deformation results obtained from a generic four-bladed, Mach scaled rotor are also presented for several different flight conditions. 


\section{PROJECTION MOIRÉ INTERFEROMETRY}

Projection Moiré Interferometry (PMI) is an optically simple, non-contacting measurement technique used since the 1970's for surface topology and shape characterization ${ }^{2}$. Widely varying applications of PMI for measuring surface topologies and deformations have ranged from orthopedics ${ }^{3}$, to aircraft components ${ }^{4}$, to animals ${ }^{5}$. The fundamentals of PMI are well $\mathrm{known}^{6,7}$, but only recently have PMI and similar systems been used to measure wind tunnel model and rotor blade deformations while under aerodynamic load. The following discussion briefly describes the fundamentals of PMI and the image processing steps used to obtain quantitative deformation profiles.

\subsection{PMI Fundamentals}

Conventional PMI relies on the projection of a grid of equispaced, parallel lines onto the surface of the object of interest. When the object in a reference condition is imaged by a CCD camera, the grid lines will have a regular spatial distribution across the object surface. Under load, the object deforms, and the projected grid lines lie in different spatial locations. Subtraction of digitized images acquired in the reference and deformed conditions produces an interferogram containing moiré fringes, which are deformation contours. This process is often referred to as projection moiré difference contouring, shown schematically in Figure 1. The moiré fringe contour interval, or fringe sensitivity constant, is determined by the projected grid line pitch and angles of projection and observation. Figure 2 shows a generic PMI configuration using symmetric illumination and observation. A Ronchi ruling, i.e. a binary grating of parallel lines having equal spacing and thickness, is used to generate the projected grid lines. The system is sensitive to the out-of-plane component of displacement, along the bisector of the angle $(\alpha+\beta)$. Geometric analysis leads to the relation ${ }^{6}$ :

$$
\Delta z=F S C=\frac{d}{\tan \alpha+\tan \beta}
$$

where $\Delta z$ is the distance between contour planes, FSC is the fringe sensitivity constant, $d$ is the distance between the projected grid lines on the object surface, and $\alpha$ and $\beta$ are the angles of illumination and observation, respectively. Nonsymmetric optical configurations are possible, but care must be taken to ensure the desired deformation component is measured and that the contour planes remain linear. The contour planes will remain linear and horizontal if the projector and receiver remain in the same horizontal plane. For these cases, equation (1) remains valid for determining the fringe sensitivity constant. This permits configurations such as normal illumination and oblique observation without introducing non-linearities in the contour planes. Further derivation for more complicated non-symmetric configurations is presented in references 2 and 6.

\subsection{PMI Image Processing}

The most recent advancements in PMI have occurred in the development of automated image processing routines for extracting quantitative deformation profiles from PMI interferograms. Although the interferogram is a valuable tool for real-time visualization of the deformed object, interferograms alone do not provide detailed quantitative results. Differences in image processing methodology distinguish PMI from the projected grid method. For the projected grid method, centroidal positions of each projected grid line were registered and tracked to obtain rotor blade deflection data. The PMI image processing steps adopt procedures from interferometric analysis to obtain quantitative displacement profiles.

Algorithms for quantitative interpretation of PMI interferograms are generally based on phase shifting. Phase shifting interprets deformations as changes in optical phase. Further processing of the phase distribution and division by the system wave number $2 \pi / F S C$ produces the reconstructed deformation profile. Phase shifting can be accomplished either (a) mechanically, by physically moving the Ronchi ruling and recording successive images, or (b) computationally, by mathematically phase shifting a computer-generated reference grid and interfering it with a digitized PMI exposure. Phase shifting by mechanical movement of the ruling can introduce errors from mechanical tolerances and requires multiple exposures of the deformed object in a stationary position. The latter requirement precludes mechanical phase shifting from rotorcraft blade deformation studies since the blades of an operating rotor are never stationary. Thus, computational phase shifting is used for the current work. 
The image processing steps used to obtain quantitative deformation profiles from digitized PMI images are shown in Figure 3 for a test specimen in a laboratory environment. The test specimen is a $250-\mathrm{mm}$ long aluminum beam, fixed at the left end, with the free end deflected out of plane. Figure 3a shows a digitized image of the beam in a deformed state. The projected grid lines are apparent. Perspective distortion caused by oblique observation of the test specimen was present, but has been removed using bilinear warping techniques ${ }^{8.9}$. The corrected image shown in Figure $3 b$ is then computationally interfered with a reference grid, Figure 3c. The computer generated reference grid is a perfect sinusoid varying in intensity along the rows of the image. The reference grid period is chosen to correspond with the mean projected grid line period observed by the CCD camera. The interference process depicted in Figure $3 \mathrm{c}$ is performed four times, each with the computational reference grid phase incremented by $90^{\circ}$. The resulting interferograms contain low frequency moiré fringes and high frequency artifacts from the interference process. After low-pass filtering, only the moiré fringes remain as shown in Figure 3d, and the wrapped phase map in Figure $3 \mathrm{e}$ is computed by ${ }^{10}$ :

$$
\theta=\tan ^{-1}\left[\frac{I_{270}-I_{90}}{I_{0}-I_{180}}\right]
$$

In equation (2) above, $\theta$ is the optical phase, $I_{x}$ are the phase shifted interferograms as in Figure $3 \mathrm{~d}$, and the subscripts represent the phase of the computer generated reference grid. The phase values shown in Figure $3 \mathrm{e}$ have been scaled such that black corresponds to $0^{\circ}$ phase, while white corresponds to $359^{\circ}$ phase. Finally, phase unwrapping must be performed on the phase distribution to obtain the surface topology of the deformed object. Phase unwrapping is the most difficult portion of the entire process, and routines to perform the operation vary widely in scope and applicability. A comprehensive review of phase unwrapping algorithms appears in reference 11. The custom algorithms used here perform unwrapping on small subsections of the image to prevent error propagation, and contain intelligence to accommodate noise and minor fringe discontinuities. Following phase unwrapping, the phase distribution is divided by the system wave number $2 \pi / F S C$ to obtain a surface topology of the deformed object. This process must be performed for the object in both its deformed and reference conditions. Differencing the two resulting surface topologies yields the quantitative deformation profile shown in Figure 3f. After performing the initial software setup, the processing time required to go from the raw data presented in Figure 3a to the actual displacement profile in Figure $3 \mathrm{f}$ is approximately 30 seconds when processed on a $166 \mathrm{MHz}$ Pentium PC. Experiments using this beam to investigate PMI system performance yielded deformation measurements accurate to within $0.1 \mathrm{~mm}$ at resolutions better than $50 \mu \mathrm{m}$. However, the resolution will decrease with increasing field-of-view.

\section{APPLICATION OF PMI TO ROTORCRAFT TESTING}

The design and implementation of a PMI system for measuring rotorcraft blade deformation presents unique challenges beyond conventional deformation studies. The system must have a large field-of-view to permit measurements over a wide azimuth range, acquire conditionally sampled azimuth dependent data, and obtain near-instantaneous images of the spinning rotor for determination of both mean and dynamic deformation. A PMI system satisfying these criteria and its use for rotorcraft blade deformation measurements is described below.

\subsection{Optical Configuration}

PMI is optically simple, requiring only two major components: a projector and receiver. The PMI projection system constructed for the Langley 14-by 22-Foot Tunnel is shown in Figure 4. The PMI system installation is shown in Figure 5. A $15 \mathrm{~W}, 800 \mathrm{~nm}$ broadband laser diode bar was used as the projector illumination source, providing high illumination energy at short pulse duration. Light emitting from the laser was collected by a cylindrical lens and directed to backilluminate a 79 line $/ \mathrm{cm}$ Ronchi ruling. The ruling was placed at the image plane of a standard $50 \mathrm{~mm}$ F/1.4 SLR camera lens, whose focus was adjusted to sharpen the grid line projection in the plane of the rotor. A $75 \mathrm{~mm}$ diameter mirror, antireflective coated for the near infrared, was positioned in front of the projector lens to direct the projected grid pattern vertically upward to illuminate the underside of the rotating blades. The nominal elevation of the blades was 2.2 meters above the projection system. The projected grid line pitch at the measurement plane was $15.75 \mathrm{~mm}$. 
The high power output of the laser diode permitted measurements to be made over a 1.2-x 1.2-meter field-of-view, covering over $50^{\circ}$ of rotor azimuth. Diode illumination levels were sufficient to capture the azimuthal rotation of the blades to within $1.4^{\circ}$ at rotor speeds of $2000 \mathrm{rpm}$. The broadband emission wavelength of the laser diode rendered speckle undetectable. Although any non-coherent light source can be used, the use of broadband laser diodes provides other operational advantages over other sources such as flash lamps. These include lights-on facility operation without loss of contrast of the PMI projected grid lines, and simultaneous operation with other laser-based instrumentation techniques via optical band pass filtering.

The PMI receiver was a generic RS- 170 analog video camera electronically shuttered to $1 / 10,000$ second. The camera was fitted with a $28-70 \mathrm{~mm}$ zoom lens and positioned to view the underside of the rotor blades. The laser diode was synchronized with the video camera to provide illumination only throughout the open shutter exposure. An IR-pass filter was installed to block visible light from the CCD array while allowing collected laser light to pass. The interlaced analog video signal was digitized in fields to 640-x 240-x 8-bits using a PC-based frame grabber. Only one field per video frame was captured and used for processing. Following camera calibration and removal of perspective and optical distortions, a spatial resolution of $1.47 \mathrm{~mm} /$ pixel was realized.

\subsection{Conditional Sampling}

Conditional sampling of the PMI receiver video signal was required to obtain blade deformation measurements at designated rotor azimuths. To implement conditional sampling for PMI and other instrumentation techniques, a custom Helicopter Rotor Azimuth Positioning System (HRAPS) was integrated with the rotor shaft encoder. The shaft encoder continuously output digital pulses at a rate of 1024 per revolution. The HRAPS counted each pulse, and latched the azimuth position when triggered. If the latched azimuth position corresponded to one of the designated positions downloaded into a lookup table, the HRAPS set a data valid line high. Other instruments monitored the sense of the data valid line to determine whether the data should be saved.

To fully implement conditional sampling with PMI, the same trigger signal was provided to HRAPS and the laser diode controller such that the HRAPS latched the shaft encoder position in coincidence with each laser pulse. Initially, video was continuously digitized into a single frame buffer at a given frame rate. The data valid line was polled after each image acquisition. If the data valid line was low, video continued to be digitized into the original frame buffer, overwriting the previous data. If the data valid line was high, video acquisition advanced to the next frame buffer, thus saving the valid data in host RAM. This cycle continued until the desired number of valid azimuth dependent images were acquired. The entire image sequence was then written to disk.

\subsection{Calibration Procedures}

Reference condition images of the test object are required for PMI. The continuously variable positions of the rotor blades make it difficult to establish a reference state for rotorcraft. Generally, the desired reference plane for rotorcraft testing is the horizontal plane at the height of the rotor hub center. Reference images of the blades at designated azimuth positions could potentially be obtained by positioning and leveling the blades within this plane. However, at flight conditions, dynamic lead-lag can inhibit reference/deformed image overlay and significantly complicate image processing. Rather than using this approach, a 1.2-x 1.2-meter aluminum honeycomb panel was supported horizontally at the height of the rotor hub center and illuminated by the PMI projector. The rigidity of the aluminum honeycomb prevented sagging in the middle of the panel when horizontally mounted. The downward facing side of the panel was painted flat white to ensure high grid line contrast. Images of the panel and projected grid lines were acquired using the PMI receiver camera and processed to establish the horizontal plane containing the honeycomb panel as the reference condition. Thus, subsequent measures of rotor blade deformation were obtained relative to this horizontal plane.

The aluminum panel was also used to perform in-situ instrument calibration. Prior experience has shown the largest systematic error source in PMI systems of this type is the ability to accurately determine the illumination and observation angles $\alpha$ and $\beta$ for determining the fringe sensitivity constant [see equation (1) and Figure 2]. Rather than calculate the fringe sensitivity constant based on measured values of $\alpha$ and $\beta$, the fringe sensitivity constant was deduced directly by vertically lifting one edge of the panel at known increments. PMI measurements were taken at each increment, and the 
slope induced across the panel was obtained. Using the slope information, the value of the fringe sensitivity constant was determined to be $19.0 \mathrm{~mm} /$ fringe.

The honeycomb panel also provided a dot card calibration target for removal of perspective and optical distortion in the acquired PMI images. A regular grid of dots was attached to the side opposite that used for the reference calibrations. The honeycomb panel dot card side was faced down to perform the perspective distortion calibration. Images of the card under laser illumination were acquired and used to determine the piecewise bilinear warping coefficients necessary to remove the optical distortions in the digitized PMI data images.

\section{EXPERIMENT}

The primary purpose of the Rotor Wake/Configuration Aerodynamics Test (RW/CAT) was to investigate the effect of the fuselage on the rotor wake geometry. This required measuring the independent and combined wakes of the rotor and fuselage to isolate non-linear interactions. The Isolated Rotor Test System (IRTS) was constructed since it is impossible to investigate isolated rotor and fuselage effects using models incorporating the rotor and fuselage about a single drive system. The IRTS system was post-mounted from the ceiling of the tunnel and permitted rotor operation either alone or with a fuselage supported underneath it. These configurations are shown in Figures 6a-b. The fuselage was lowered to the test section floor when the rotor was tested in the isolated configuration. The rotor system consisted of a Mach-scaled, four bladed, fully articulated rotor with a rotor radius of $860 \mathrm{~mm}$. These research blades were not aeroelastically scaled, but rather very stiff in flapwise, chordwise, and torsional bending. The blades had a rectangular planform, -8 degrees of linear twist, and a NACA 0012 airfoil along the span. The blades and their properties are documented in reference 12 and a detailed description of the hub can be found in reference 13.

Conventional laser velocimetry (LV) and Doppler Global Velocimetry (DGV) ${ }^{14}$ measurements of flowfield velocity were acquired to map the rotor wake geometry. The rotor wake geometry and vortex strength are largely affected by blade position and deformation. Therefore, a complete database of wake geometry must include blade position and deformation data. To measure the blade deformation profiles, the PMI system described in section 3 was installed in the floor of the facility as shown in Figure 5. The PMI projector was positioned at approximately $50 \%$ rotor radius on the blade-advancing side of the helicopter, projecting vertically to illuminate the underside of the rotating blades. A 1-x 1-meter floor opening near tunnel centerline (Figure 5) provided ample optical access for the PMI projector. This opening also housed the third component of the LV system used for aerodynamic velocity measurements. No modifications were made to the existing LV hardware for the incorporation of the PMI system. The PMI receiver camera was mounted at the same streamwise location and height as the projector, 2.3 meters outboard perpendicular to tunnel centerline. A single floor panel was removed to accommodate the camera and provide optical access. The PMI and DGV systems were synchronized and operated simultaneously to provide near instantaneous blade deformation / wake velocity correlations for several flight conditions. Unification of the two instruments required the synchronization of 9 video cameras, 5 data acquisition computers, and two lasers. This represents the first known attempt at using unified global instrumentation techniques to measure dynamic blade deformation and aerodynamic reaction ${ }^{15}$.

A typical data point was acquired using the following procedure. After daily calibrations and model inspections, the tunnel test section was secured and the rotor was operated in hover. Tunnel speed was then increased to the desired operating condition as model parameters were set simultaneously. Upon reaching steady state, data were acquired by the tunnel static data system, DGV data acquisition system, and PMI data acquisition system. The DGV and PMI systems acquired instantaneous, conditionally sampled azimuth-dependent data for blade \#1 azimuth positions of $0^{\circ}$ to $90^{\circ}$ in $10^{\circ}$ increments. The azimuth $(\Psi)$ and blade number convention is shown in Figure 7. These azimuths were chosen to maximize the amount of unsteady blade deformation data obtained while minimizing data storage requirements. PMI measurements were obtained for two different blades: blade \#4 for $\Psi=90^{\circ}$ to $110^{\circ}$ ( $\Psi=0^{\circ}$ to $20^{\circ}$ for blade \#1), and blade $\# 1$ for $\Psi=70^{\circ}$ to $90^{\circ}$. PMI data for blade \#1 azimuth positions of $30^{\circ}$ to $60^{\circ}$ were not processed since the blades, in majority, were out of the PMI receiver field-of-view. Approximately 30 images were acquired at each azimuth for each flight condition. Figure 8 shows an example PMI data image viewing the underside of the blade and identifies the blade geometry. Note the image is rotated $90^{\circ}$, with the tunnel ceiling corresponding to the right side of the image. The projected grid lines required to form moiré fringes are readily apparent. Creation of the moire patterns and determination 
of the quantitative deformation profiles is performed computationally. The PMI data were processed off-line following completion of the image acquisition.

\section{RESULTS}

PMI measurements were acquired for a variety of test conditions, both with the isolated rotor and with the fuselage in place. Table 1 contains tunnel velocity and rotor parameters for the seven flight conditions discussed here. For brevity of discussion, the phrase "condition 418 " will be abbreviated $\mathrm{C} 418$ and likewise for the other conditions. The range of forward speeds from $9.1 \mathrm{~m} / \mathrm{s}$ to $41.6 \mathrm{~m} / \mathrm{s}$ covers the helicopter flight envelope from very low speed operations to moderate forward speeds, while the thrust coefficient range from 0.004 to 0.008 simulates thrust levels from $1 \mathrm{~g}$ to maneuver thrust values. Figure 9 presents quantitative rotor blade deformation profiles for blade $\# 4$ at $\Psi=0^{\circ}$ at the seven different test conditions summarized in Table 1. The fuselage was in the lowered position for the data shown in Figure 9. The reason for the approximate $4^{\circ}$ offset in the blade rotation direction remains to be fully explained, but could be the combined result of (a) blade lead-lag, (b) shaft encoder offset errors, or (c) PMI receiver viewing angle. This $4^{\circ}$ offset is consistent among other azimuth locations measured. The deformation profiles on the left side of the chart are measured with respect to a horizontal plane aligned vertically with the center of rotation of the rotor hub. Profiles on the right side of the chart indicate the change in blade deformation as a function of test condition with respect to C418. Changes in blade shape can be clearly observed. Figure 9 also illustrates the ability of the PMI technique to measure deformation over the entire blade.

Pixels corresponding to the $25 \%$ chord location have been extracted from the PMI processed data and plotted as a function of span in Figures 10 and 11 to further illustrate the quantitative effects of flight condition on the blade deformation profile. The PMI data have been anchored to a value of $0 \mathrm{~mm}$ at a point $23 \%$ rotor radius at $25 \%$ chord, corresponding to the edge of the rotor cuff attachment. This is necessary since the PMI system measures relative surface displacements in its current configuration. The data shown represent the mean blade profiles for blade $\# 4$ at $\Psi=90^{\circ}$, lowered fuselage. As advance ratio increases from 0.05 to 0.23 in Figure 10, the deflection of the blade decreases from the zero reference point by approximately $4 \mathrm{~mm}$ at the tip. The flight condition change is a change in forward speed for a fixed thrust condition of the rotor system. Each blade is effectively working harder at the two lower flight conditions, as the speeds are below the minimum power speed. Because the rotor hub is fully articulated, the inboard flapping hinge allows the blades to come to an equilibrium position with respect to the lift and centrifugal forces on the blade. For the lower flight speeds, the blades are coned higher and deflected greater with respect to the hinge. In each case, the rotor blade is shown to be deflecting linearly along the spanwise direction, indicating little flapwise bending other than the steady-state coning deflection. For these flight conditions, the PMI data shows trends which seem to be reasonable and correct.

The effect of varying the rotor thrust or blade loading for a constant forward flight speed is presented in Figure 11 . In this figure, C419 is a thrust level well below a normal scaled operating condition, and C418 increases thrust to a nominal scaled thrust. In Figure 11 it can be clearly seen that blade 4 deflects significantly (at least $10 \mathrm{~mm}$ ) in the change from C419 to C418. As in the analysis for Figure 10, this change is related to the balance of forces around the flapping hinge, and the flight condition for $\mathrm{C} 419$ is such that the low rotor lift does not create very much steady state coning. As thrust is increased from $\mathrm{C} 419$ to $\mathrm{C} 418$, the blade loading for the low speed flight condition increases and the blades cone upwards. PMI measures a deflection which corresponds to a coning change of about $0.5^{\circ}$. Calculations of the expected steady state coning change between the two conditions is $0.5^{\circ}$, indicating that PMI is measuring the correct deflection.

The results shown in Figure 12 show the effects of the fuselage on blade deflection. Moving the fuselage up and down beneath the rotor system only slightly changed the cyclic control settings of the rotor system, where the integrated rotor thrust and forward speed were held constant. Although the blades were free to adjust their position relative to the flapping hinge, the fuselage effects are mostly upwash effects fore and aft on the rotor disk ${ }^{16}$, and not much change in deflection is observed at the $90^{\circ}$ azimuth measurement location. The deflection of the blade relative to the reference point was constant for conditions with either the isolated rotor or the rotor/fuselage combination. Fuselage effects on blade unsteadiness are shown in Figure 13 for C418 and C425. The PMI system noise floor is also plotted in Figure 13, determined by calculating single pixel statistics from an ensemble of images of the aluminum honeycomb calibration panel. The fact that the revolution-to-revolution RMS deflection fluctuations are above the PMI system noise floor indicates the fluctuations are blade induced and not an instrumentation artifact. For the very low speed flight condition C418, it is evident that the flow environment is turbulent and thus causes blade $\# 4$ to fluctuate up to $1 \mathrm{~mm}$ when the fuselage is in the lowered position. 
The fluctuation decreases as the fuselage is raised beneath the rotor. The fuselage locally accelerates the flow, which can modify the turbulent flowfield producing decreased blade unsteadiness.

The indexing of the rotor blades and acquisition trigger cycles enabled data to be obtained for both blades 1 and 4 at $90^{\circ}$ azimuth. This allows the rather interesting blade-to-blade comparison shown in Figures 14 and 15 . Figure 14 shows that although the blades were of the same design, blade 1 consistently flew higher than blade 4 . There are several possible explanations. When testing rotor models, the blades are usually tracked in a hover condition; this means the blades are adjusted such that the blade tips fly within the same plane, or track. For this model, the blades are usually considered within the tracking tolerance if the tips are tracking within $5 \%$ chord of each other. This equates to $5 \mathrm{~mm}$ for this blade set. The difference in the blade deflection measured by the PMI system for the blades is less than the $5 \mathrm{~mm}$ tracking tolerance, so a probable explanation for the blade-to-blade difference is the rotor track tolerance was greater than the resolution of the PMI measurements. Figure 15 shows the revolution-to-revolution RMS fluctuations between blades 1 and 4 at $\Psi=90^{\circ}$. The difference in RMS fluctuation is probably related to manufacturing differences in the blade composite layup structure. Each of the blades experiences the high fluctuations for $\mathrm{C} 418$ and the fluctuations decrease for the higher speed C425. Figure 14 has shown the mean deflection is approximately the same for the blades, and the flowfield each blade is experiencing should be almost exactly the same since these data were acquired in the same data set. The difference in the RMS fluctuation can only be explained by blade-to-blade differences which the PMI technique is sensitive enough to detect.

The PMI deflection data were interrogated for the displacement of the leading and trailing edge of the blade chord along the span of the blade. These deflections were converted into a chordwise angle, or twist, at each spanwise station. Figure 16 shows the measured twist along the span of the blade. The blades were designed and fabricated with a built-in linear twist of 8 degrees nose down referenced from the center of rotation to the tip. Figure 16 shows that the PMI measurements were able to capture the twist of the blades within 1 degree which is considered good. The PMI measurements are hampered in this area by the small chord of the blades. The measurement error and fluctuations are exaggerated in the twist computation since a small change in deflection will have a large change in effective angle over a small blade chord. PMI was able to distinguish the changes in collective angle setting of the blades for other flight conditions and the PMI measurements appeared to correlate well with the rotor control system measurements of changes in rotor blade pitch.

The azimuth-dependent nature of blade deflection is shown in Figure 17. Blade tip deflection data at $r / R=1.0$ is plotted versus azimuth for $\mathrm{C} 418, \mathrm{C} 419$, and $\mathrm{C} 425$, fuselage down. While tip deflections for $\mathrm{C} 418$ and $\mathrm{C} 419$ remain relatively constant as a function of azimuth, $\mathrm{C} 425$ exhibits a downward trend attributed to the $-3^{\circ}$ rotor shaft pitch setting for this flight condition. This azimuth dependence is clearly demonstrated in the PMI measured blade deflection profiles shown in Figure 18. An ensemble of conditionally sampled, azimuth dependent PMI data were assembled to construct the figure. Figure 18a shows dewarped PMI data images for each conditionally sampled azimuth location, and the resulting quantitative displacement profiles have been overlayed in Figure $18 \mathrm{~b}$.

\section{ACCURACY AND RESOLUTION}

This experiment was a first attempt at using PMI for measuring dynamic rotor blade deflections. Since this type of data has never been acquired for the hub and blades used in this test, there is no standard to compare against for determination of absolute accuracy. Previous laboratory experience has shown the largest systematic error in PMI measurements lies in the ability to accurately determine the angles of illumination and observation. These errors were minimized by performing in-situ calibration of the instrument to determine the fringe sensitivity constant [FSC, equation (1)] as described in Section 3.3. Calibrations of the fringe sensitivity constant agreed to within $3 \%$, establishing the best case absolute accuracy of the measurements. Using data from the in-situ calibrations, the PMI system noise floor was determined to be $0.08 \mathrm{~mm}$ based on single pixel statistics. This noise floor defines the instrument's minimum deformation resolution. Most of the data exhibits slight undulations along the spanwise profiles. These are most likely image processing artifacts, as the blades are too stiff to develop higher order bending modes at the flight conditions tested. Work is continuing to diagnose the causes of these artifacts. 


\section{CONCLUSIONS}

This initial proof-of-concept test has demonstrated the capability of Projection Moiré Interferometry (PMI) to acquire accurate, full field rotorcraft blade deformation data. These are believed to be the first measurements of their kind providing near-instantaneous, quantitative, whole field blade deformation profiles conditionally sampled as a function of rotor azimuth. The near-instantaneous nature of the measurements permitted examination of both mean and time varying statistics such as blade unsteadiness. The results obtained show changes in blade deflection, twist, and dynamic behavior with varying operating conditions. Measurement accuracy is believed to be within $3 \%$, with $0.08 \mathrm{~mm}$ deformation resolution. The simultaneous application of PMI and Doppler Global Velocimetry (DGV) was successfully performed and represents the first attempt at using unified global instrumentation techniques to measure dynamic blade deformation and aerodynamic reaction. PMI development will continue for both independent operation and operation as part of a unified instrumentation system to measure the rotor wake and blade deformation in large scale wind tunnels.

\section{ACKNOWLEDGEMENTS}

The authors appreciate the support of the members of the Measurement Science and Technology Branch, the Rotorcraft Aerodynamics Group, and the 14- by 22-Foot Subsonic Tunnel staff.

\section{REFERENCES}

1. Mercker, E., Pengel, K., Kube, R., Van der Wall, B., Boutier, A., Micheli, F., "On the Blade Deformation Measured at a Scaled Helicopter Rotor", Proceedings on Aeromechanics Technology and Product Design, Bridgeport, CT, October 11-13, 1995.

2. Patorski, K., Handbook of the Moiré Fringe Technique, Elsevier Science Publishers, 1993. pp. vii - xi.

3. Kurz, H., and Leder, O., "PC-based moiré for field studies on the human body surface", Proc. SPIE 1395, pp. 1066 $1073,1990$.

4. Dykes, B.C., Proceedings of the Fourth International Conference on Stress Analysis, Cambridge, 125. April, 1970.

5. Miles, C.A., and Speight, B.S., "Recording the shape of animals by a moiré method", Journal of Physics E: Scientific Instruments, Vol. 8, pp. 773 - 776, 1975.

6. Pirodda, L., "Shadow and projection moiré techniques for absolute or relative mapping of surface shapes", Optical Engineering 21(4), pp. 640 - 649, July/August 1982.

7. Selected Papers on Optical Moiré and Applications, SPIE Milestone Series, Vol. MS 64, 1992. Indebetouw, G., and Czarnek, R., editors.

8. Phillips, D., "Image Processing in C, Part 14: Warping and Morphing", C/C++ Users Journal, pp. 55 - 68, October, 1995.

9. Meyers, J.F., "Doppler Global Velocimetry - The Next Generation?" AIAA $17^{\text {th }}$ Aerospace Ground Testing Conference, paper AIAA-92-3897, Nashville, TN, July 6-8 1992.

10. Patorski, K., Handbook of the Moiré Fringe Technique, Elsevier Science Publishers, 1993. pp. 372-373.

11. Robinson, D.W., "Phase Unwrapping Methods", Interferogram Analysis, D.W. Robinson and G.T. Reid editors. Inst. of Phys. Pub., Bristol, 1993. pp 194 - 229.

12. Berry, J. D.; and, May, M. J.: Flapping Inertia for Selected Rotor Blades. NASA TM 104125, AVSCOM TR 91-B-019, November, 1991.

13. Phelps, A. E.,III; and, Berry, J. D.: Description of the U. S. Army Small-Scale 2-Meter Rotor Test System. NASA TM 87762, AVSCOM TM-86-B-4, February, 1987.

14. Meyers, J.F., "Development of Doppler Global Velocimetry as a Flow Diagnostics Tool", Measurement in Fluids and Combustion Systems, Special Issue, Measurement Science and Technology, 6(6), pp. 769 - 783, June 1995.

15. Meyers, J.F., Lee, J.W., Fletcher, M.T., and South, B., "Hardening Doppler Global Velocimetry Systems for Large Wind Tunnel Applications", Presented at the $20^{\text {th }}$ AIAA Advanced Measurement and Ground Testing Technology Conference, Albuquerque, NM, June 15-18, 1998.

16. Berry, J. D.; and, Althoff, S. L.: Computing Induced Velocity Perturbations Due to a Helicopter Fuselage in a Free Stream. NASA TM 4113, AVSCOM TR 89-B-001, June, 1989. 


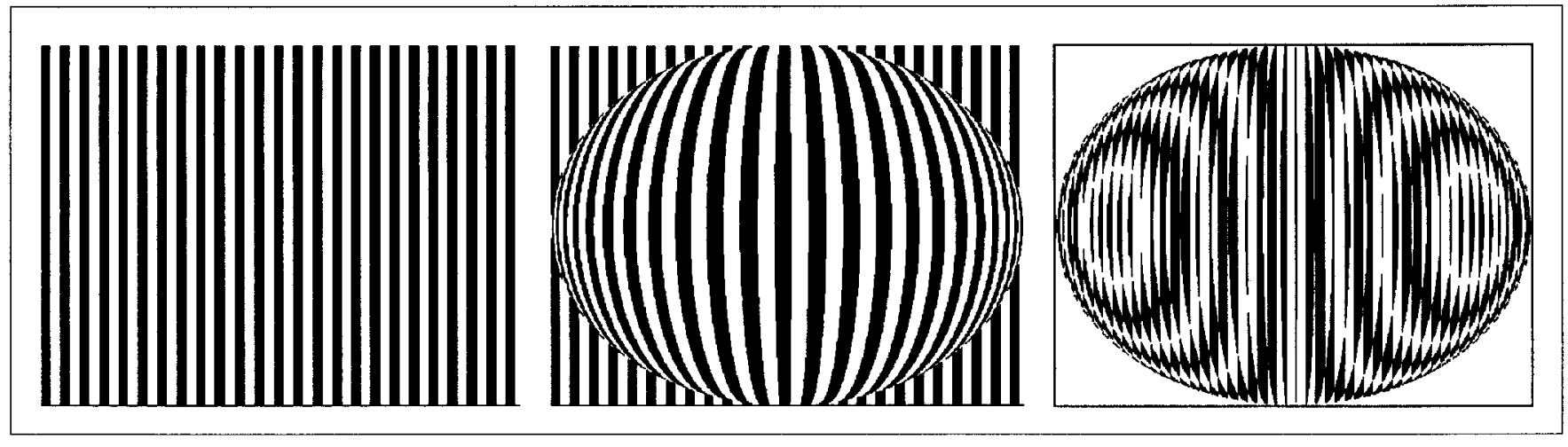

\section{Reference State - Deformed State $=$ Deformation Contours}

Figure 1: Projection moiré difference contouring.

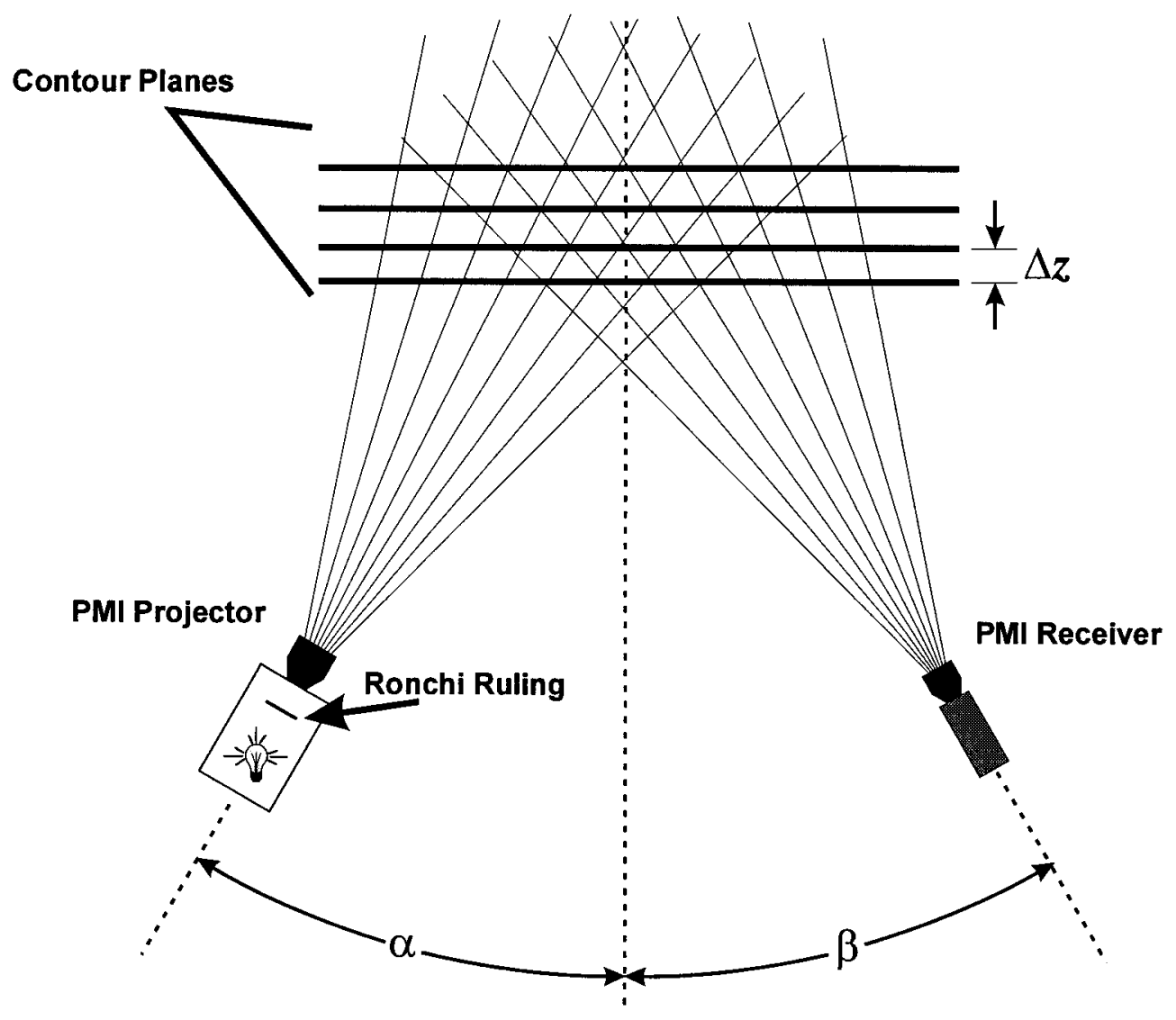

Figure 2: Symmetric projection moiré interferometry configuration. 


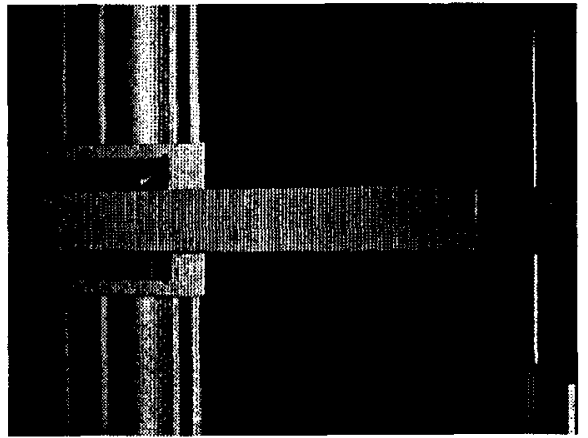

(a) Raw Data

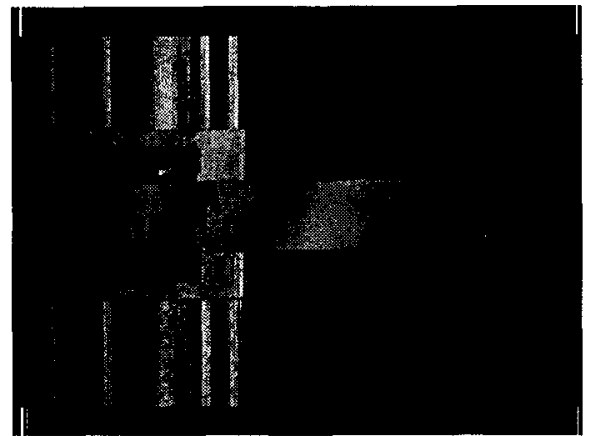

(d) Low-pass Filtered

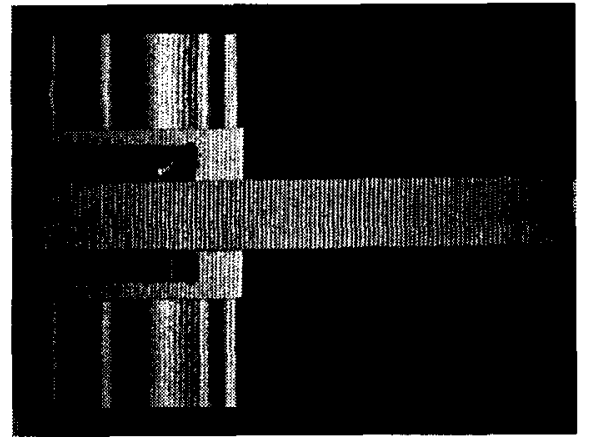

(b) De-warped Image

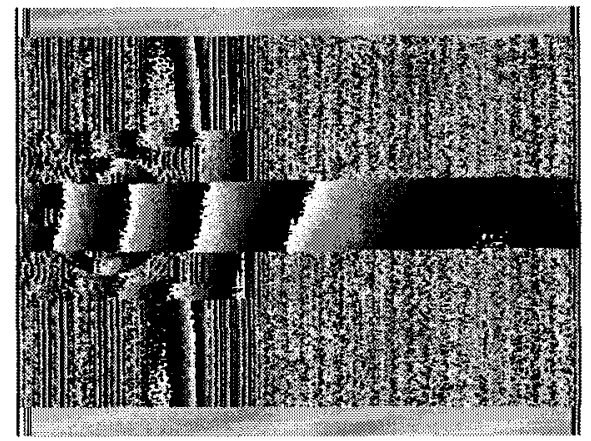

(e) Wrapped Phase Map

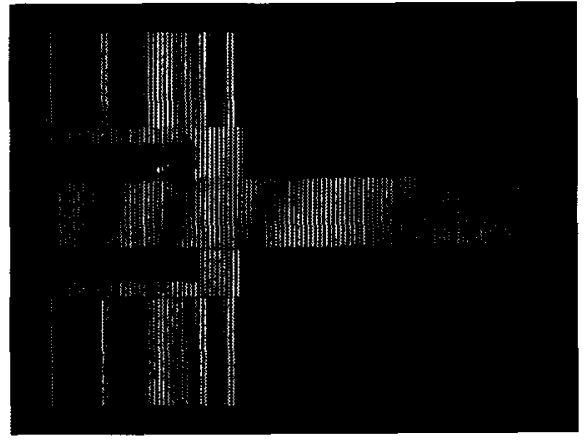

(c) Interfered with Reference

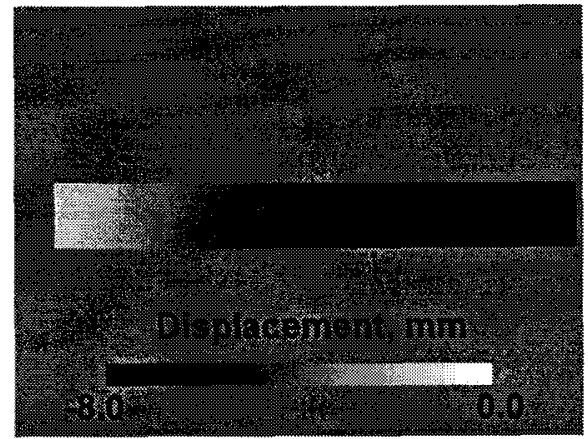

(f) Displacement Profile

Figure 3: PMI image processing steps.

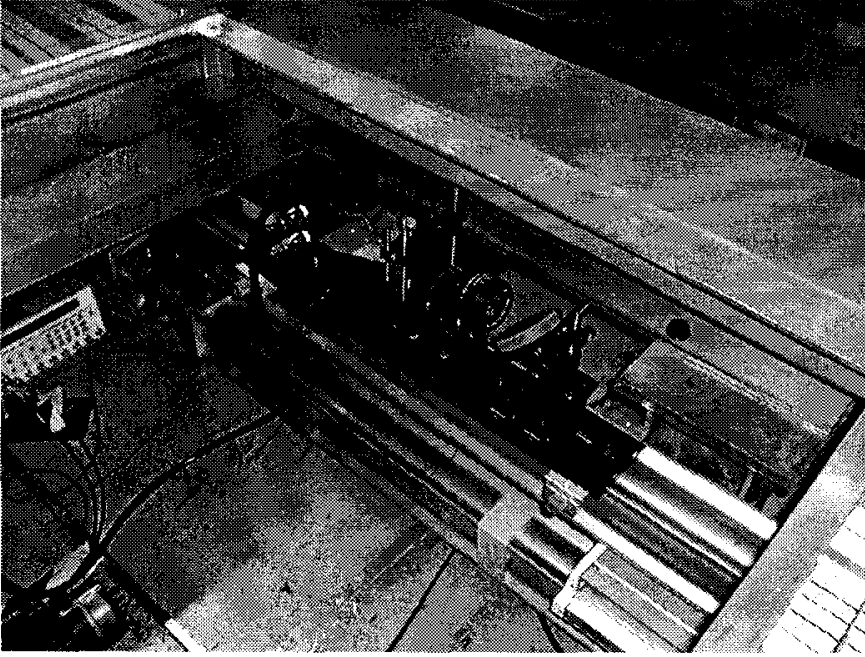

Figure 4: PMI projection system for rotorcraft blade deformation measurements.

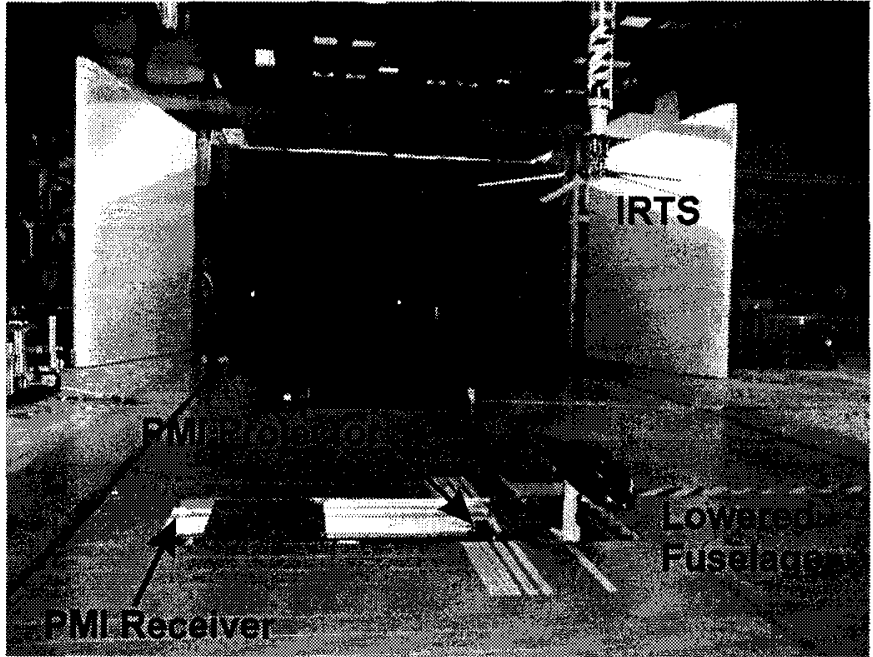

Figure 5: PMI system installed in the 14-By 22-Foot Subsonic Tunnel for the Rotor Wake / Configuration Aerodynamics Test. 


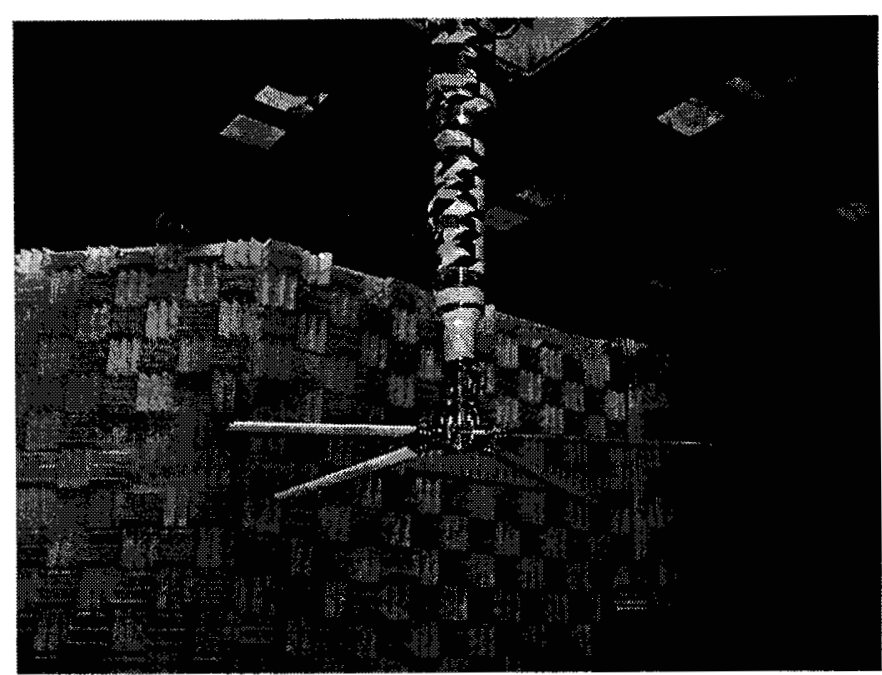

(a)

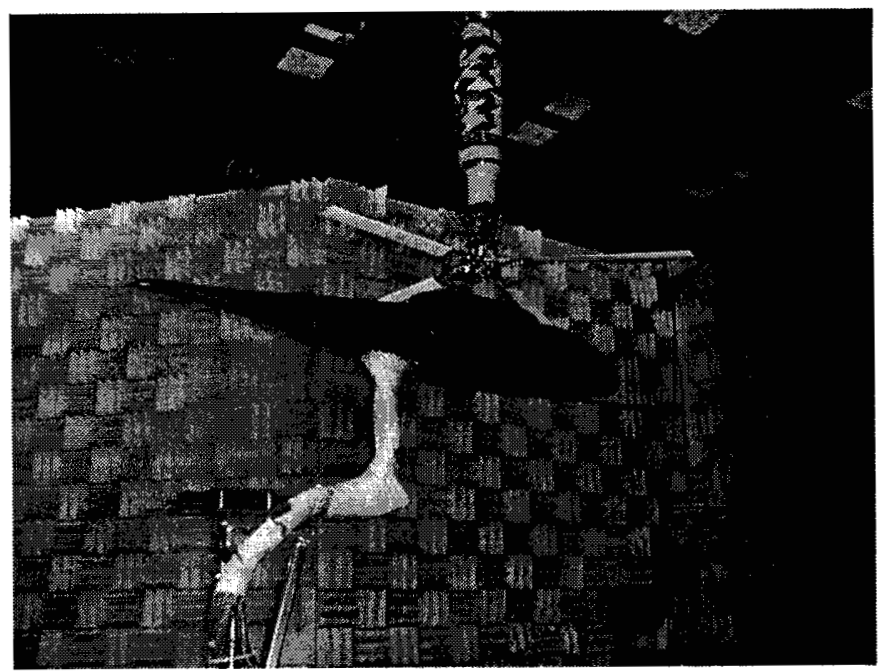

(b)

Figures 6a-b: (a) Isolated Rotor Test System, IRTS, and (b) IRTS + fuselage.

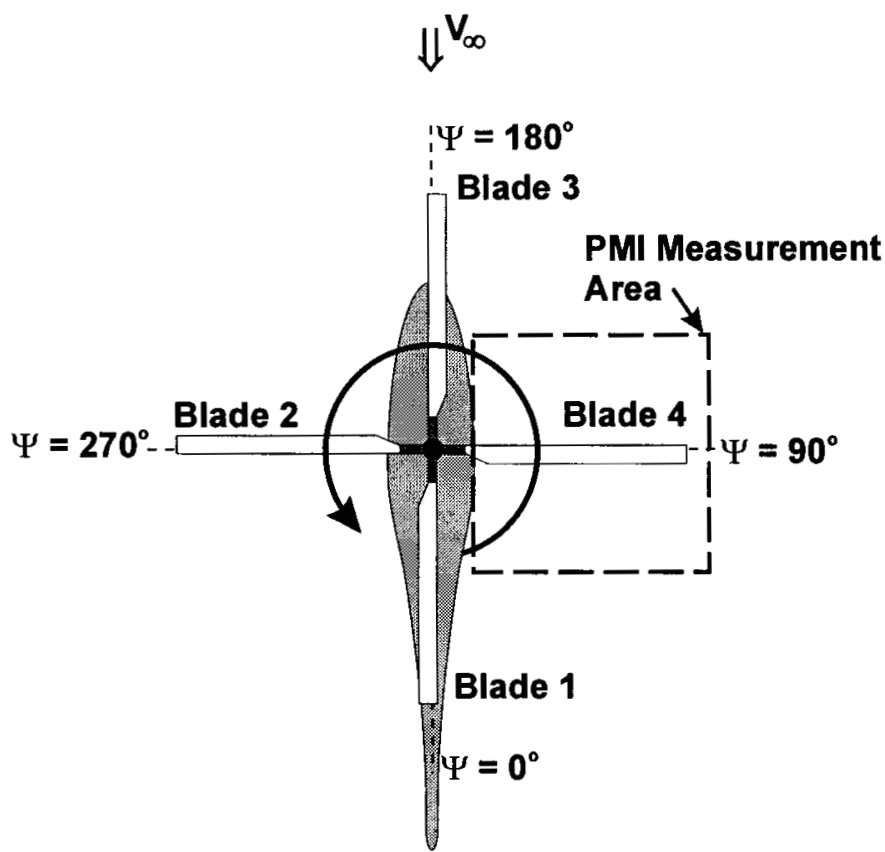

Figure 7: Rotor azimuth and blade numbering convention. The PMI measurement area is also illustrated.

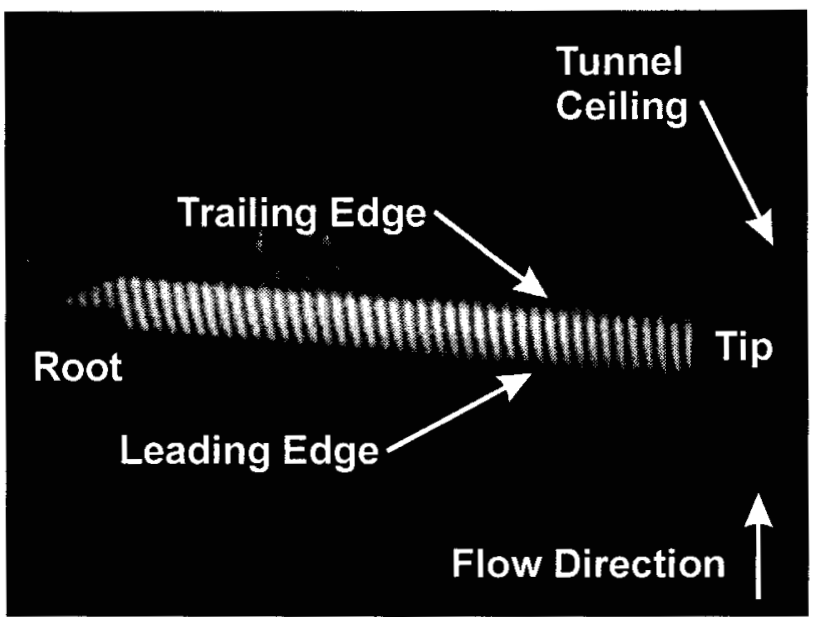

Figure 8: Rotor blade geometry within PMI images. 
Table 1: Rotor and tunnel conditions corresponding to the seven flight conditions addressed in this paper. Rotor speed was $2000 \mathrm{rpm}$ for all cases.

\begin{tabular}{cccccc}
\hline \hline Condition & $\begin{array}{c}\text { Dynamic Press. } \\
\text { Q (Pa) }\end{array}$ & $\begin{array}{c}\text { Free Stream } \\
\text { Velocity, } \mathbf{m} / \mathbf{s}\end{array}$ & $\begin{array}{c}\text { Rotor } \\
\text { Advance Ratio }\end{array}$ & $\begin{array}{c}\text { Rotor Shaft } \\
\text { Angle, deg. }\end{array}$ & $\begin{array}{c}\text { Rotor Thrust } \\
\text { Coefficient }\end{array}$ \\
\hline \hline 418 & 47.9 & 9.1 & 0.05 & 0 & 0.0064 \\
419 & 47.9 & 9.1 & 0.05 & 0 & 0.0040 \\
423 & 47.9 & 9.1 & 0.05 & 0 & 0.0080 \\
424 & 478.7 & 27.1 & 0.15 & 0 & 0.0064 \\
425 & 478.7 & 27.1 & 0.15 & -3 & 0.0064 \\
430 & 478.7 & 27.1 & 0.15 & -3 & 0.0080 \\
431 & 1100.1 & 41.6 & 0.23 & -3 & 0.0064 \\
\hline \hline
\end{tabular}

\section{Flight Condition \\ Blade Deformation Profile

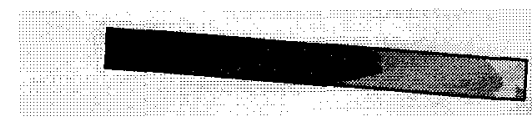

423
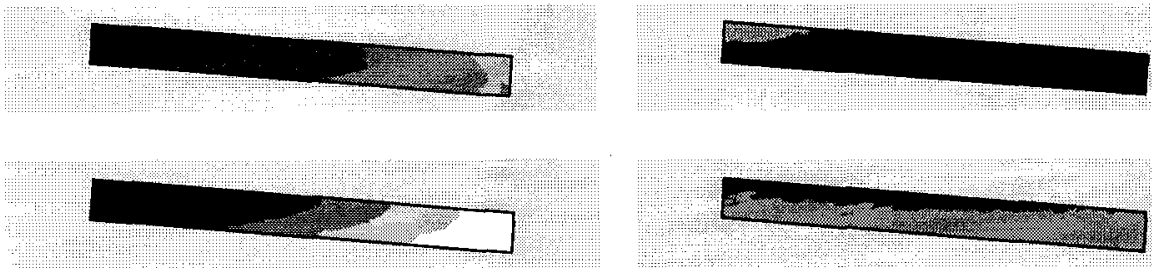

424
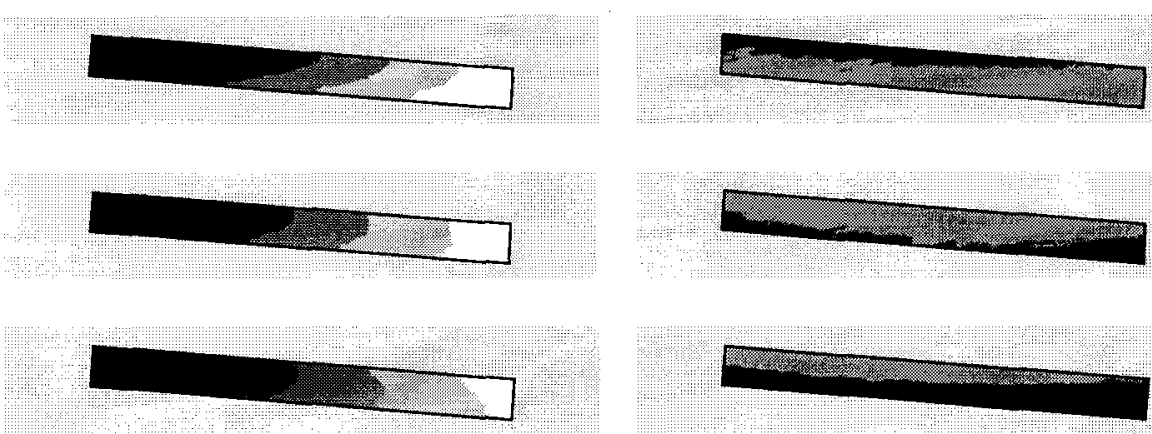

425

430

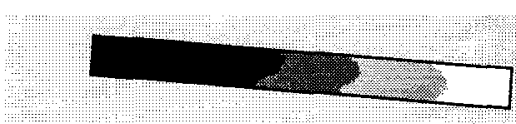

431
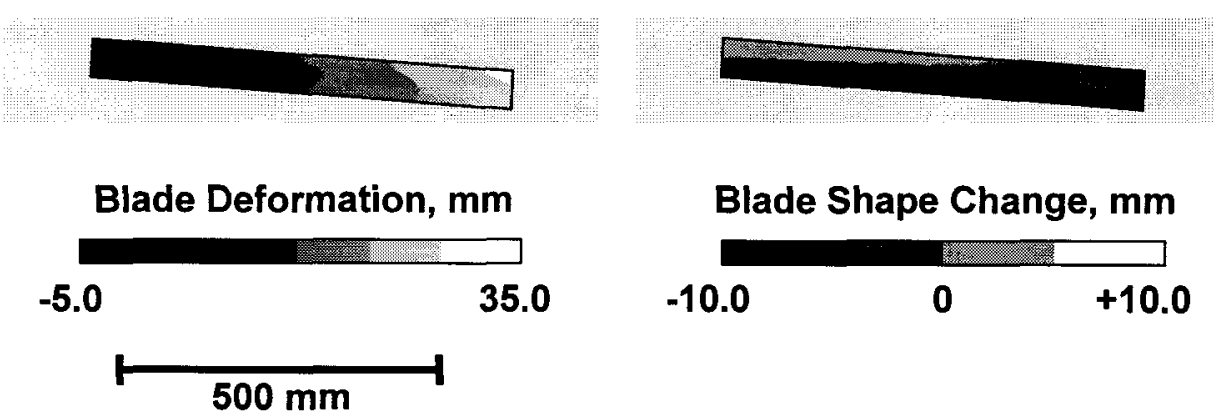

Figure 9: PMI measured blade deflection profiles for seven different flight conditions, blade $\# 4, \Psi=90^{\circ}$. 


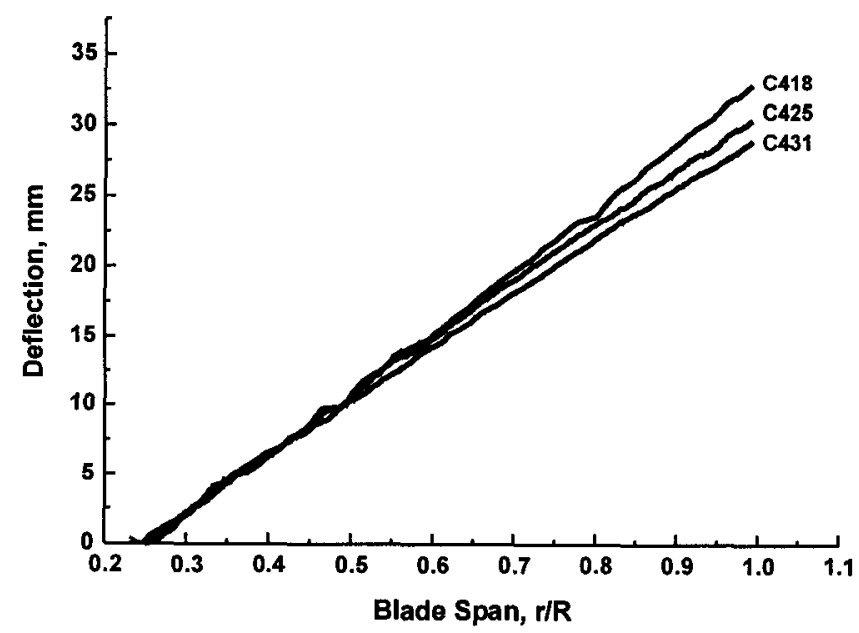

Figure 10: Effect of rotor advance ratio on blade deflection for blade $\# 4,25 \%$ chord, $\Psi=90^{\circ}$.

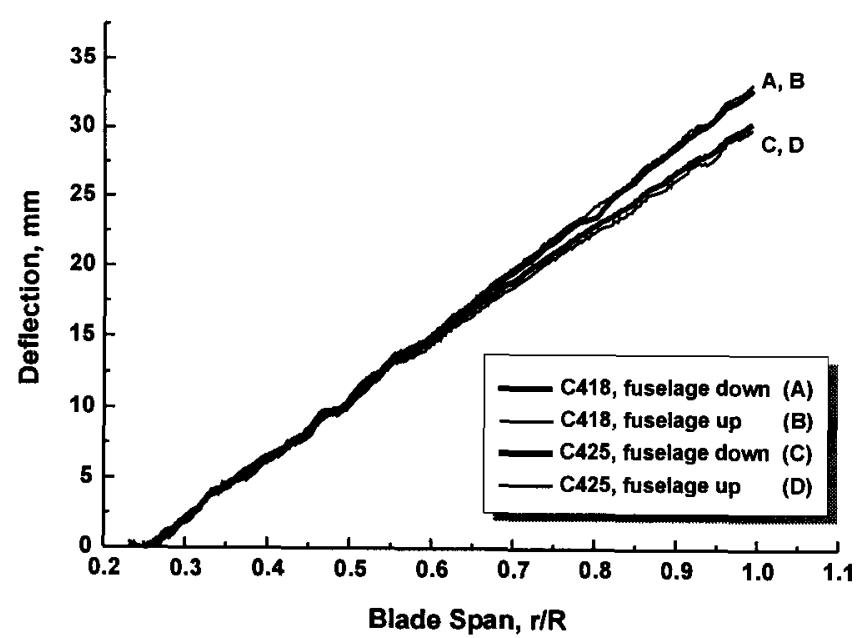

Figure 12: Fuselage effects on mean blade deflection for blade $\# 4,25 \%$ chord, $\Psi=90^{\circ}$.

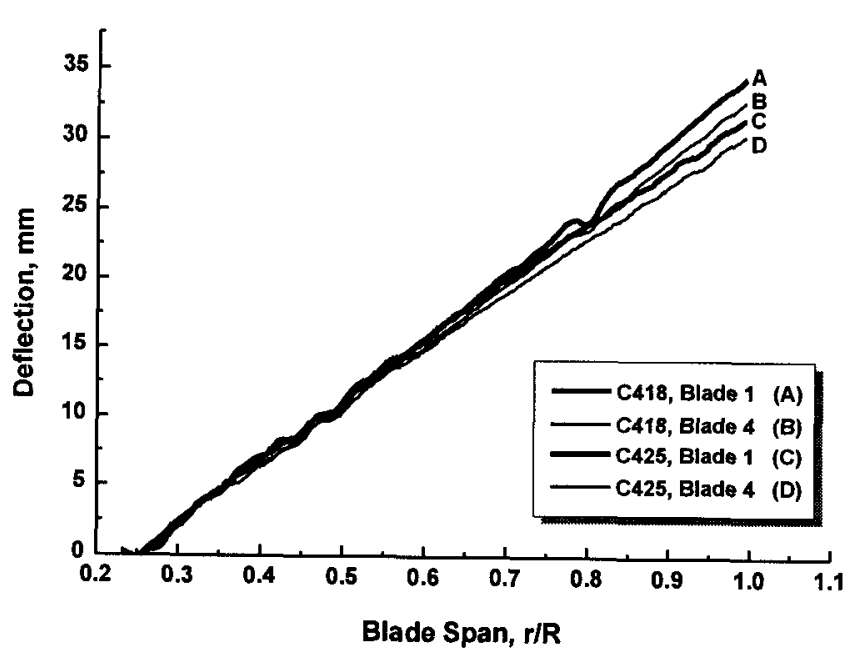

Figure 14: Blade-to-blade comparison, mean deflection profiles at $25 \%$ chord, $\Psi=90^{\circ}$.

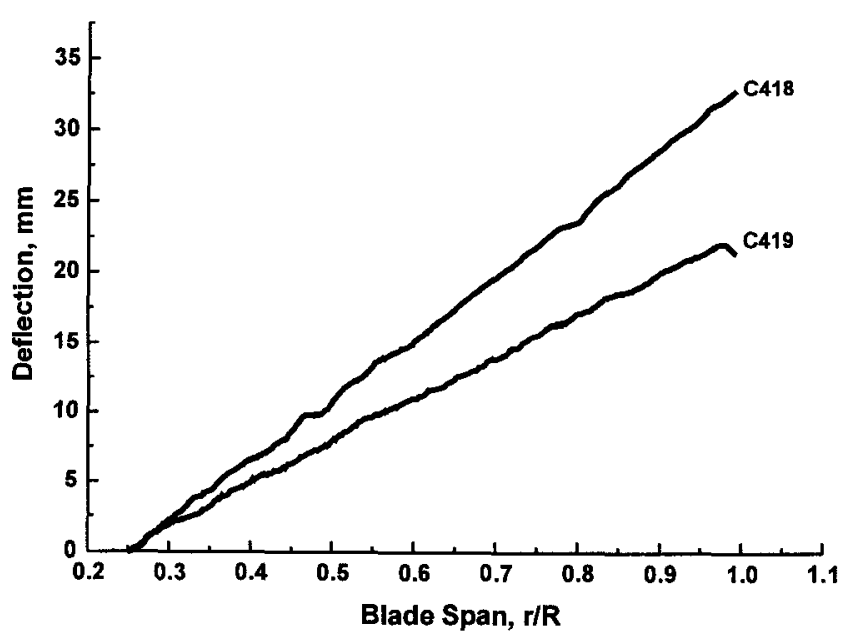

Figure 11: Effect of rotor thrust coefficient on blade deflection for blade $\# 4,25 \%$ chord, $\Psi=90^{\circ}$.

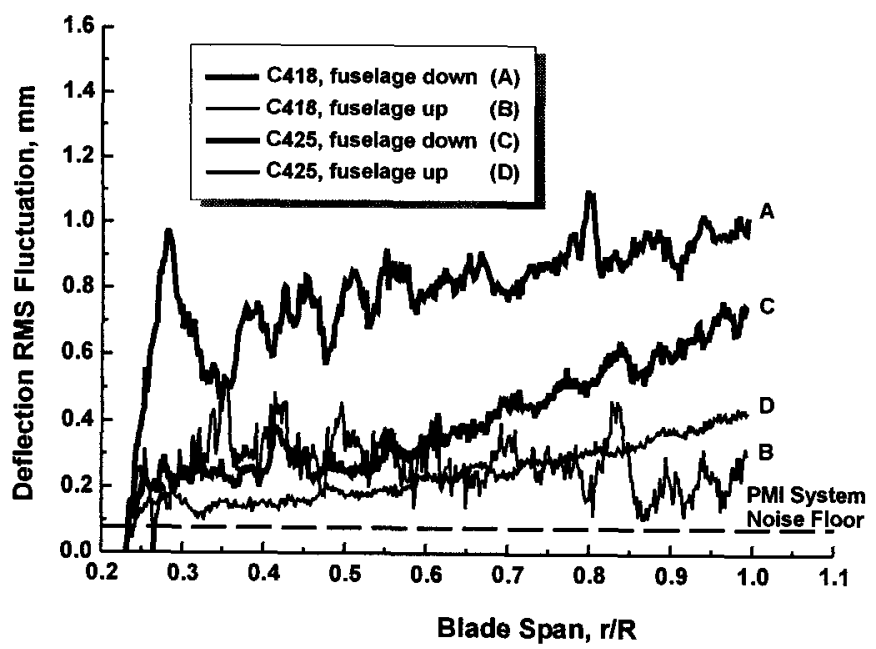

Figure 13: Fuselage effects on blade dynamic deflection for blade $\# 4,25 \%$ chord, $\Psi=90^{\circ}$.

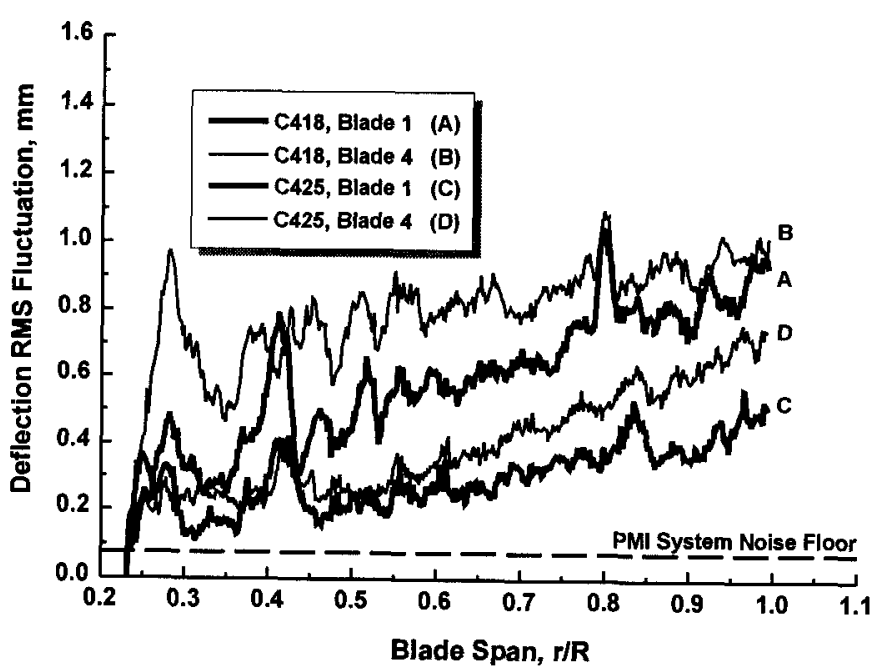

Figure 15: Blade-to-blade comparison, dynamic blade deflection at $25 \%$ chord, $\Psi=90^{\circ}$. 


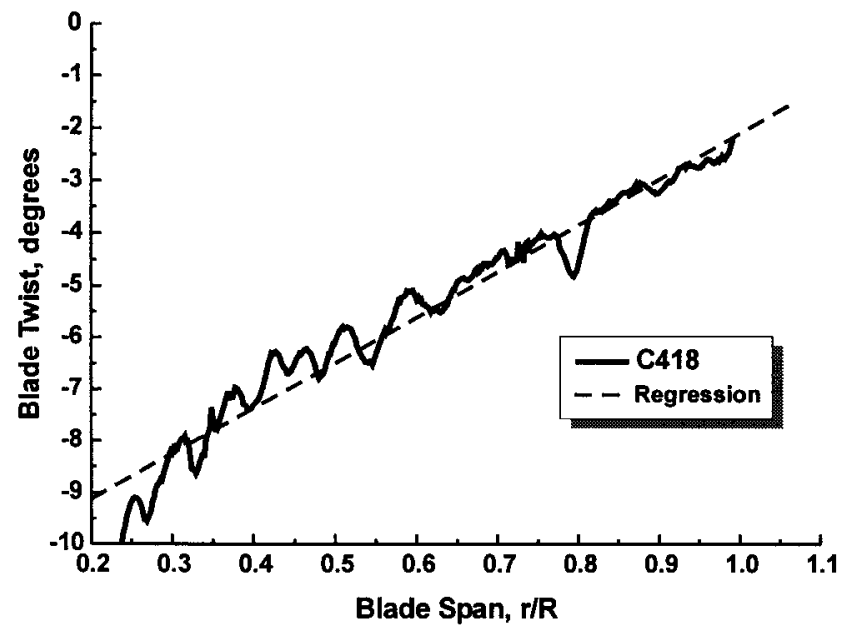

Figure 16: Spanwise blade twist distribution, blade \#4, $\Psi=90^{\circ}$. Regression slope $=0.0102 \mathrm{deg} . / \mathrm{mm}$.

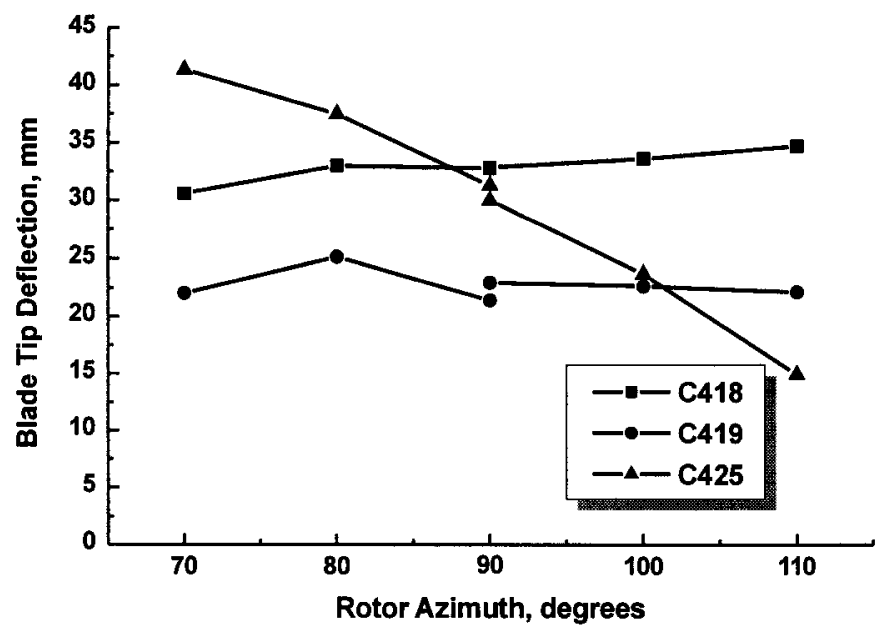

Figure 17: Azimuth dependent mean blade tip deflection, $\mathrm{r} / \mathrm{R}=1.0$. Rotor shaft angle for $\mathrm{C} 425$ was set to -3 degrees.

\section{(a) Dewarped PMI Data}

\section{(b) Blade Deflection Profiles}

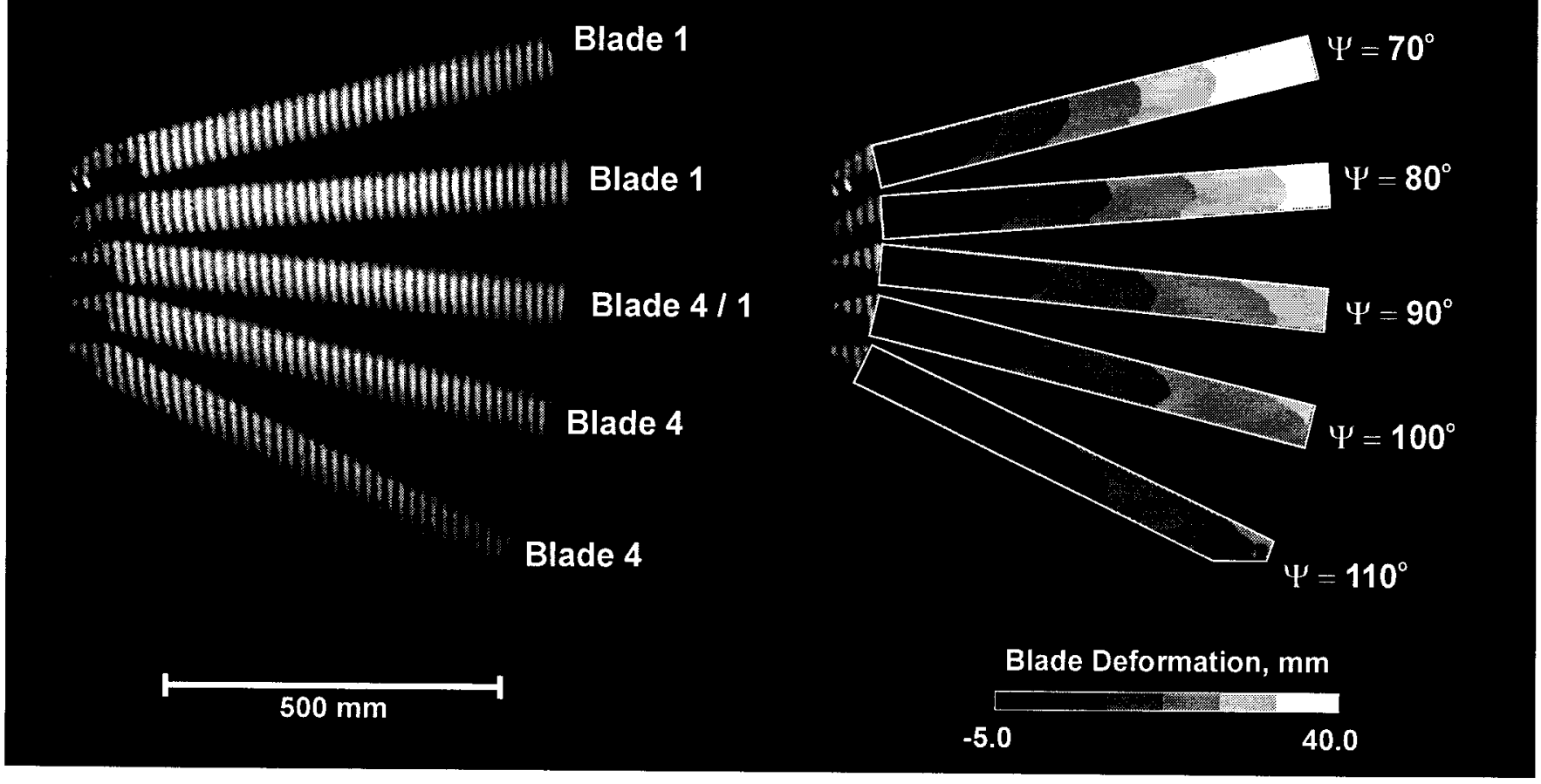

Figure 18: Azimuth dependent PMI data and measured blade deformation profiles, C425, fuselage down. Rotor shaft angle was set to -3 degrees. 